\title{
Función pulmonar y calidad de vida en niños y adolescentes con bronquiolitis obliterante por adenovirus
}

\author{
STEFHANIE NAYAR E.*, MÓNICA SAAVEDRA B.*, \\ ANA MARÍA ESCOBAR C.* y ALBERTO VIDAL G.*
}

Pulmonary function and quality of life in children and adolescents with bronchiolitis obliterans post-adenoviral infection

Introduction: Adenovirus infection is an important cause of pneumonia in Chilean children. Postinfectious Bronchiolitis Obliterans (PIBO) is the most important complication. There are few studies assessing pulmonary function and quality of life in PIBO patients. Objective: The aim of this study is to assess the pulmonary function and the quality of life of patients with PIBO and the correlation between both variables. Methods: 14 children with PIBO in follow up at the pediatric pulmonology outpatient clinic of a public children hospital were included in this study. Study period: April 2009 - April 2010. Pulmonary function was assessed in a medical visit by spirometry, flow/volume curve and intrathoracic gas volume measurement. The following indices were analyzed FVC, FEV $V_{1}, F E F_{25-75}, F E V_{1} / F V C, R V$, $T L C, R V$ and RV/TLC. A survey of a self-administered Quality of Life (PedsQL, version 4.0, Spanish for Chile) was applied at the visit to investigate their global, physical and psychosocial quality of life. The Pearson linear correlation between quality of life and the pulmonary function test parameters was assessed, in the statistical analysis a p value $<0.05$ was considered significant. Results: The mean age of our patients was 12.4 (range: 9-19 years-old). The functional alterations were characteristics of an obstructive respiratory disorder in $64.3 \%$ of the patients. Average pulmonary function test indices showed decreases in $F E V_{1}$ (75\%), $F E F_{25-75}$ (48.8\%), FEV $/ F V C$ (67.6\%) with normal value of FVC (93,7\%) as well as pronounced increases in RV (333.5\%), TLC (156\%) and RV/TLC (51.8\%). Mean change of $\mathrm{FEV}_{1}$ and $\mathrm{FEF}_{25-75}$ post-bronchodilator was $11.3 \%$ and $26.4 \%$ respectively. Only $14.3 \%$ of our patients showed normal pulmonary function. The quality of life parameters were in average $58.2 \%$ of overall quality of life, $58.6 \%$ of physical quality of life and $57.9 \%$ of psychosocial quality of life. We found a positive correlation between global quality of life and $F E V_{1}, F V C$ and $F E F_{25-75}$ (correlation index 0.54, 0.53 and 0.53 respectively) as well between physical quality of life and $F E V_{1}, F V C, F E V_{1}$ / $F V C$ and $F_{E F} F_{25-75}$ (correlation index 0.86, 0.82, 0.72 and 0.55 respectively). Conclusions: Most patients with PIBO showed pulmonary function impairment characterized by an obstructive respiratory pattern. Better quality of life correlates with better values in spirometric parameters.

Key words: Pulmonary function, quality of life, postinfectious bronchiolitis obliterans, adenovirus.

\section{Resumen}

Introducción: La infección por adenovirus es una causa importante de neumonía en niños chilenos. La bronquiolitis obliterante (BOPI) es la complicación más importante. Existen pocos estudios donde evalúen la función pulmonar y la calidad de vida de los pacientes con BOPI. Objetivo: Evaluar la función pulmonar y la calidad de vida de los pacientes con BOPI y la correlación entre ambas variables. Métodos: Se incluyeron 14 niños con BOPI en el policlínico de la unidad de broncopulmonar de un hospital público de niños. Período de estudio: abril de 2009-abril de 2010. La función pulmonar se evaluó en una visita médica por espirometría, curva flujo / volumen y la determinación de volumen de gas intratorácico. Los índices analizados fueron los siguientes CVF, VEF $, F E F_{25-75}, V E F_{1} / C V F$,

* Unidad Brocopulmonar, Hospital Roberto del Río. 
VR,CPT, VR y VR / CPT. En la misma visita se realizó una encuesta auto-administrada de Calidad de Vida (PedsQL, versión 4.0, español de Chile) para investigar la calidad de vida global, física y psicosocial. Se aplicó la correlación lineal de Pearson entre calidad de vida y los parámetros de función pulmonar. Se consideró estadísticamente significativo un $p<0,05$. Resultados: La edad media de nuestros pacientes fue de 12,4 (rango: 9-19 años). La alteración funcional característica fue la obstructiva en el 64,3\% de los pacientes. El promedio de los índices de pruebas de función pulmonar mostraron una disminución en $V E F_{1}(75 \%), F_{25} F_{25-75}$ (48,8\%), $V E F_{1} / C V F(67,6 \%)$ con un valor normal de la CVF (93,7\%), así como aumentos pronunciados en VR (333,5\%), CPT (156\%) y VR/CPT (51,8\%). La media del cambio del $V E F_{1}$ y $\mathrm{FEF}_{25-75}$ post-broncodilatador fue 11,3\% y 26,4\% respectivamente. Sólo el 14,3\% mostró una función pulmonar normal. Los parámetros de calidad de vida fueron en promedio 58,2\% de calidad de vida general, 58,6\% de calidad de vida física y el 57,9\% de calidad de vida psicosocial. Se encontró una correlación positiva entre la calidad de vida global y $V E F_{1}$, CVF y FEF 25-75 (índice de correlación de 0,54, 0,53 y 0,53, respectivamente), así como entre calidad de vida física y VEF $C V F, V E F_{1} / C V F$ y $F_{E F} F_{25-75}$ (índice de correlación de 0,86, 0,82, 0,72 y 0,55 respectivamente). Conclusiones: La mayoría de los pacientes con BOPI mostraron un deterioro de la función pulmonar de tipo obstructivo con atrapamiento aéreo. Mejor calidad de vida se correlaciona con mejores valores en los parámetros de la espirometría.

Palabras clave: Función pulmonar, calidad de vida, bronquiolitis obliterante, adenovirus.

\section{Introducción}

El adenovirus (ADV) es la tercera causa de infección respiratoria aguda baja en niños que requieren hospitalización en Chile y una causa importante de secuelas a corto y largo plazo, entre las que destaca la bronquiolitis obliterante (BOPI) $)^{1-7,8,12,14}$.

En nuestro hospital se ha realizado una vigilancia epidemiológica desde 1989 y hasta el presente año, detectándose ADV en infecciones respiratorias agudas bajas adquiridas en la comunidad con frecuencias que oscilan entre 3 y $20 \%{ }^{7}$.

La mayoría de los pacientes que cursa con una infección por ADV se recupera satisfactoriamente, pero los que presentan una infección severa, evolucionan con una elevada morbilidad y mortalidad ${ }^{7,8}$.

La Bronquiolitis Obliterante es una enfermedad pulmonar crónica que resulta como complicación de injuria pulmonar severa, en este caso secundario a una infección por ADV, en la cual se observa una inflamación de los bronquiolos pulmonares, causando una obstrucción crónica de los mismos. Clínicamente se caracteriza por la presencia de tos, disnea, sibilancias y crépitos persistentes y alteración de la función pulmonar, sin respuesta o con respuesta parcial a broncodilatadores ${ }^{9-11}$.

Las pruebas de función pulmonar de niños con BOPI generalmente muestran obstrucción severa al flujo aéreo fija, con disminución de la distensibilidad y aumento de la resistencia, con una pequeña respuesta a broncodilatador ${ }^{10-12}$.

Los volúmenes pulmonares típicamente muestran una mayor capacidad pulmonar total y aumento de la relación VR/CPT, en consonancia con la hiperinsuflación y atrapamiento aéreo ${ }^{10,11}$. La mayoría de los pacientes con BOPI, requiere oxigeno suplementario hasta un año después del alta hospitalaria y presenta reingresos frecuentes por descompensaciones respiratorias, que disminuyen en los años siguientes ${ }^{14,15}$. Otro hallazgo frecuente en niños con BOPI es la limitación en la realización de ejercicio físico ${ }^{16}$.

Existe escasa investigación en relación a la calidad de vida de estos pacientes. La Organización Mundial de la Salud (OMS) definió la calidad de vida (CV) como la percepción personal de un individuo de su situación en la vida, dentro del contexto cultural y de los valores en que vive y relación con sus objetivos, expectativas y valores e intereses, afirmando que la CV no es igual a estado de salud, estilo de vida, satisfacción con la vida, estado mental ni bienestar, sino que es un concepto multidimensional que debe tener en cuenta la percepción por parte del individuo de éste y otros conceptos de la vida ${ }^{17}$. El término calidad de vida relacionada con la salud (CVRS), designa los juicios de valor que un paciente realiza acerca de las consecuencias de su enfermedad o su tratamiento ${ }^{18}$. La evaluación de calidad de vida debe realizarse a través de cuestionarios que estén validados para la población en estudio, tomando en cuenta su edad, lenguaje y cultura entre otros ${ }^{17}$. Nosotros creemos que la calidad de vida de los pacientes portadores de bronquiolitis obliterante secuela de una infección grave por adenovirus en la infancia está alterada y que esta alteración está relacionada con el compromiso en la función pulmonar. 


\section{Método}

Durante el período comprendido entre abril de 2009 y abril de 2010 se citó telefónicamente a pacientes entre 8 y 19 años, portadores de BOPI controlados periódicamente en policlínico de broncopulmonar del hospital Roberto del Río, o que se encontraban en el registro electrónico del hospital. Se excluyó a los pacientes que presentaban otra enfermedad pulmonar crónica conocida como fibrosis quística y displasia broncopulmonar y a los pacientes cuyos padres o tutores no accedieron a participar del estudio a través de la firma de un consentimiento informado.

En esta cita se aplicó la encuesta de calidad de vida autoadministrable PedsQL, versión 4.0 ${ }^{19}$ para niños entre 8 y 12 años y para adolescentes de 13 a 18 años, traducida para Chile por la empresa que diseñó la encuesta y validada en español ${ }^{20}$. La encuesta se compone de 23 preguntas que comprenden 4 dimensiones de la calidad de vida, 8 preguntas de funcionamiento físico, 5 de funcionamiento emocional, 5 de funcionamiento social y 5 de funcionamiento escolar. Siguiendo las guías de administración de los autores de estos cuestionarios ${ }^{19,20}$, con respuestas estratificadas de 0 a 4 (0: nunca, 1: casi nunca, 2: a veces, 3: con frecuencia, 4: siempre), luego estas respuestas se expresaron en porcentajes (0: $0 \%$, 1:25\%, 2: 50\%, 3: 75\%, 4: 100\%), y se calcula un promedio de los porcentajes en cada dimensión, y posteriormente se obtiene un promedio

Tabla 1. Promedios de índices espirométricos y volúmenes pulmonares en 14 pacientes portadores de bronquitis obliterante por adenovirus

\begin{tabular}{|lc|}
\hline Indices espirométricos & $\mathbf{\%}$ \\
$\mathrm{CVF}$ & 93,7 \\
$\mathrm{VEF}_{1}$ & 75,0 \\
$\mathrm{FEF}_{25-75}$ & 48,8 \\
$\mathrm{VEF}_{1} / \mathrm{CVF} \cdot 100$ & 67,6 \\
\hline Volúmenes pulmonares & Litros \\
$\mathrm{CPT}$ & 5,5 \\
$\mathrm{VR}$ & 2,8 \\
$\mathrm{CRF}$ & 3,7 \\
$\mathrm{VR} / \mathrm{CPT} \cdot 100$ & $51,8 \%$ \\
\hline
\end{tabular}

Indices espirométricos (en \% de su valor teórico): CVF: capacidad vital forzada; $\mathrm{VEF}_{1}$ : volumen espiratorio forzado al primer segundo, $\mathrm{FEF}_{25-75}$ : flujo espiratorio forzado entre el 25 y 75\% de la CVF. Volúmenes pulmonares (en litros, BTPS): CPT: capacidad pulmonar total, VR: volumen residual, CRF: capacidad residual funcional. de la dimensión escolar, emocional y social para adquirir el puntaje psicosocial. Para calcular el puntaje global se promedian el puntaje psicosocial y físico.

Durante la misma visita se realizó una espirometría y medición de volúmenes pulmonares, las que fueron realizadas por un médico broncopulmonar empleando un espirómetro Jaeger modelo MS-PFT y para la medición de volúmenes pulmonares se utilizó la técnica de barrido de helio. Los resultados obtenidos se expresaron como porcentaje de valores predictivos de Knudson y Zapletal. Para la realización e interpretación del estudio funcional se siguieron las recomendaciones de consensos nacionales e internacionales (2125). Posteriormente se calculó la correlación lineal de Pearson entre los valores promedios de calidad de vida y los índices de función pulmonar $\left(\mathrm{VEF}_{1}\right.$, CVF, $\mathrm{VEF}_{1} / \mathrm{CVF}, \mathrm{FEF}_{25-75}, \mathrm{VR}, \mathrm{CPT}, \mathrm{VR} / \mathrm{CPT}$ ). Se consideró como significativo un $\mathrm{p}<0,05$.

\section{Resultados}

Completaron la evaluación 14 pacientes entre 9 y 19 años con un promedio de edad de 12,4 años. Ocho hombres y seis mujeres. En la evaluación espirométrica se obtuvo un patrón espirométrico obstructivo en $64,3 \%$, mixto en $21,4 \%$, normal en $14,3 \%$ y restrictivo en $0 \%$ de los pacientes. Los promedios de los índices espirométricos se presentan en la Tabla 1. El cambio promedio post-broncodilatador del $\mathrm{VEF}_{1}$ y del $\mathrm{FEF}_{25-75}$ fue 11,3 y $26,4 \%$ respectivamente. En la medición de volúmenes pulmonares los promedios fueron los siguientes: VR 2,8 L (333,5\%), CPT 5,5 L (156,4\%), CRF 3,7 L, relación VR/ CPT 51,8\%. Respecto a las encuestas el promedio de calidad de vida general (CaVG) fue de $58,2 \%$, calidad de vida física (CaVF) de 58,6\%, calidad de vida psicosocial (CaVP) de 57,9\%. (Tabla 2). La dimensión más afectada fue la escolar y la

Tabla 2. Promedios de calidad de vida por dimensiones en 14 pacientes portadores de bronquitis obliterante por adenovirus

\begin{tabular}{|lc|}
\hline & $\mathbf{\%}$ \\
\hline Dimensión Emocional & 61,8 \\
+ Dimensión Social & 59,6 \\
+ Dimensión Escolar & 52,1 \\
= Puntaje Psicosocial & 57,9 \\
+ Puntaje Físico & 58,6 \\
= Puntaje Global & 58,2 \\
\hline
\end{tabular}


Tabla 3. Correlación de Pearson entre calidad de vida y función pulmonar en 14 pacientes con bronquilitis obliterante por adenovirus

\begin{tabular}{|llcccccccc|}
\hline & \multicolumn{3}{c}{ Índices espirométricos } & \multicolumn{4}{c}{ Volúmenes pulmonares } \\
Calidad de vida & $\mathbf{V E F}_{\mathbf{1}}$ & $\mathbf{C V F}$ & $\mathbf{V E F}_{\mathbf{1}} / \mathbf{C V F}$ & $\mathbf{F E F}_{25-75}$ & $\mathbf{C P T}$ & $\mathbf{V R}$ & $\mathbf{V R / C P T}$ & $\mathbf{C R F}$ \\
General & $0,54^{*}$ & $0,53^{*}$ & 0,42 & $0,53^{*}$ & $0,46^{*}$ & 0,3 & $-0,32$ & 0,37 \\
Psico-social & 0,25 & 0,27 & 0,16 & 0,30 & 0,43 & 0,3 & $-0,27$ & 0,38 \\
Física & $0,86^{*}$ & $0,82 *$ & $0,72 *$ & $0,55^{*}$ & 0,34 & 0,19 & $-0,16$ & 0,24 \\
\hline
\end{tabular}

Cada cifra representa el valor del índice de correlación lineal de Pearson. ${ }^{*} \mathrm{p}<0,05$. Indices espirométricos: $\mathrm{VEF}_{1}$ : volumen espiratorio forzado al primer segundo, CVF: capacidad vital forzada, $\mathrm{VEF}_{1} / \mathrm{CVF}$; $\mathrm{FEF}_{25-75}$ : flujo espiratorio forzado entre el 25 y 75\% de la CVF. Volúmenes pulmonares: CPT: capacidad pulmonar total, VR: volumen residual, CRF: capacidad residual funcional.

mejor evaluada por niños y adolescentes fue la emocional.

Al calcular el índice de correlación encontramos una correlación positiva entre CaVG y los valores espirométricos $\mathrm{VEF}_{1}, \mathrm{CVF}$ y $\mathrm{FEF}_{25-75}$ (Tabla 3). También fue positiva la correlación entre $\mathrm{CaVF}$ y los valores de $\mathrm{VEF}_{1}, \mathrm{CVF}, \mathrm{VEF}_{1} /$ CVF y FEF $25-75$. No encontramos una correlación significativa entre parámetros de calidad de vida y volúmenes pulmonares. La calidad de vida psicosocial no mostró relación con valores espirométricos ni de volúmenes pulmonares (Tabla 3).

\section{Comentarios}

Nuestros resultados concuerdan con otras publicaciones de BOPI, ya que en la mayoría de nuestros pacientes predominó la alteración funcional de tipo obstructiva con importante atrapamiento aéreo y escasa o nula respuesta a broncodilatadores ${ }^{2,14,25}$. Existen varias publicaciones en que se describen las secuelas funcionales y radiológicas de pacientes con $\mathrm{BOP} \mathrm{I}^{11,12,13,26}$, pero son escasas las que evalúan el compromiso en su calidad de vida. Nuestro estudio es uno de los primeros en que se demuestra objetivamente que los pacientes con BOPI muestran alteraciones en su calidad de vida. Dentro de nuestros hallazgos, encontramos que la dimensión más afectada es la escolar, lo que se explica por repetidas inasistencias en relación a controles médicos y mal estado de salud, luego le sigue la dimensión social que se ve afectada en relación a la imposibilidad de seguir el ritmo de sus compañeros o no lograr hacer sus mismas actividades, pero no está afectada por la posibilidad de entablar relaciones de amistad. La dimensión emocional es la mejor evaluada por los niños y adolescentes.

El aumento de la calidad de vida general se correlaciona con valores espirométricos más elevados probablemente por la menor alteración de calidad de vida física. No se encontró relación entre la calidad de vida psicosocial con valores espirométricos ni volúmenes pulmonares, lo que nos señala que esta dimensión no tendría que ver del todo con las limitaciones físicas y que es buena a pesar de las restricciones que estos pacientes pueden presentar. Destacamos que la calidad de vida física tiene una correlación elevada y estadísticamente significativa con los índices espirométricos, por lo que podemos concluir que los niños o adolescentes mayores de 8 años con bronquiolitis obliterante tienen mayor compromiso de su calidad de vida física en la medida que sus valores espirométricos son menores. Respecto a los volúmenes pulmonares no se logró encontrar correlación significativa con la calidad de vida, pero se encontró una correlación inversa débil aunque no estadísticamente significativa de calidad de vida con la relación VR/CPT.

Entre las limitaciones de nuestro trabajo debemos mencionar el bajo número de pacientes, que podría estar influyendo en la detección de correlaciones significativas con otras variables espirométricas o de volúmenes pulmonares. Por otro lado también debemos considerar que la medición de volúmenes se realizó por lavado de helio, método que nos da resultados menos exactos que si se hubiera realizado esta medición por medio de pletismografía. Entre las proyecciones de nuestro trabajo está realizar la evaluación de la función pulmonar con test de marcha e intentar aumentar el número de pacientes.

La importancia de nuestro trabajo está en que se establece que la calidad de vida de los pacientes portadores de bronquiolitis obliterante secuela de una infección grave por adenovirus en la infancia está alterada, principalmente por las limitaciones físicas y que el grado de alteración de la calidad de vida se relaciona con el compromiso en la función pulmonar. 


\section{Bibliografía}

1.- AVENDAÑO LF, LARRAÑAGA C, PALOMINO M A, GAGGERO A, MONTALDO G, SUÁREZ M, et al. Community and hospital-acquired respiratory syncytial virus infection in Chile. Pediatr Infect Dis J 1991; 10: 564-8.

2.- WU E, MARTÍNEZ V, ÁLVAREZ A, LARRAÑAGA C, VELA H. Casos fatales de infección por adenovirus. Rev Chil Pediatr 1990; 61: 177-84.

3.- SINGH-NAZ N, BROWN M, GANESHANANTHAN M. Nosoco-mial adenovirus infection: Molecular epidemiology of an outbreak. Pediatr Infect Dis J 1993; 12: 922-5.

4.- HORWITZ M. Adenoviruses. En: Fields BN, Knipe DM, et al., eds. Virology. 2nd Ed. New York: Raven Press, 1990.

5.- LARRAÑAGA C, KAJON A, VILLAGRA E, AVENDAÑO L F. Adenovirus surveillance on children hospitalized for acute lower respiratory infections in Chile (1988-1996). J Med Virol 2000; 60: 342-6.

6.- ADRIAN T H, WADELL G, HIERHOLZER J, WIGNAD R. DNA restriction analysis of adenovirus prototypes 1 to 41. Arch Virol 1986; 91: 277-90.

7.- $\quad$ PALOMINO M A, LARRAÑAGA C, AVENDAÑO L F. Hospital-acquired adenovirus $7 \mathrm{~h}$ infantile respiratory infection in Chile. Pediatr Infect Dis J 2000; 19: 527-31.

8.- PALOMINO M A, LARRANAGA C, VILLAGRA E, CAMACHO J, AVENDANO L F. Adenovirus and Respiratory Sincytial virus-adenovirus mixed acute lower respiratory infections in Chilean infants. Pediatr Infec Dis J 2004; 23: 337-41.

9.- ZHANG L, IRION K, KOZAKEWICH H, REIS L, CAMARGO J J. Clinical course of postinfectious bronchiolitis obliterans.Pediatr Pulmonol 2000; 29: 341-51.

10.- MOONNUMAKAL S P, FAN L L. Bronchiolitis obliterans in children. Curr Opin Pediatr 2008; 20: 272-8.

11.- AGUERRE V, CASTAÑOS C, PENA H G, GRENOVILLE M, MURTAGH P. Postinfectious bronchiolitis obliterans in children: Clinical and pulmonary function findings. Pediatr Pulmonol 2010, 45: 1180-5.

12.- TEPER A M, KOFMAN C D, MAFFEY A F, VIDAURRETA S M Lung function in infants with chronic pulmonary disease after severe adenoviral illness. J Pediatr 1999; 134: 730-3.

13.- MATTIELLO R, MALLOL J, FISCHER G. Pulmonary function in children and adolescents with postinfectious bronchiolitis obliterans. J Bras Pneumol 2010; 36: 4539.

14.- COLOM A J, TEPER A M. Post-infectious bronchiolitis obliterans. Arch Argent Pediatr 2009, 107: 160-7.

15.- CASTRO-RODRÍGUEZ J A, DASZENIES C, GARCÍA M, MEYER R, GONZALES R. Adenovirus pneumonia in infants and factors for developing bronchiolitis obliterans: A 5-year follow-up. Pediatric Pulmonology. 2006, 41: 947-53.
16.- CHANG AB, MASEL J P, MASTERS B. Postinfectius Bronchiolitis Obliterans; Clinical, radiological and pulmonary function sequelae. Pediatric Radiol 1998; 28 : 23-9.

17.- WORLD HEALTH ORGANIZATION Division of Mental Health. Quality of life assessment an annotated bibliography compiled by Louisa Hubanks and Willem Kuy Ken. WHO/MNH/ PSF 94.1, Geneva World Health Organization 1994.

18.- ESPINOSA DE LOS MONTEROS M, DE MIGUEL J, GRUPO DE TRABAJO DE ASMA DE NEUMOMADRID. Calidad de vida relacionada con la salud. Aplicación en el asma. Rev Patol Respir 2001; 1: 15-8.

19.- VARNI J W. Scaling and scoring of the Pediatric Quality of Life Inventory ${ }^{\mathrm{TM}}$ PedsQL ${ }^{\mathrm{TM}}$ http://www.pedsql.org

20.- ROIZEN M, RODRÍGUEZ S, BAUER G, MEDIN G, BEVILACQUA S, VARNI J W, et al. Initial validation of the Argentinean Spanish version of the PedsQL ${ }^{\text {TM }} 4.0$ Generic Core Scales in children and adolescents with chronic diseases: acceptability and comprehensibility in low-income settings. Health Qual Life Outcomes 2008, 6: 59.

21.- MILLER M, CRAPO R, HANKINSON J, BRUSASCO V, BURGOS F, CASABURI R, et al. General considerations for lung function testing. Eur Respir J 2005; 26 : 153-61.

22.- MILLER M, HANKINSON J, BRUSASCO V, BURGOS F, CASABURI R, COATES A, et al. Standardisation of spirometry. Eur Respir J 2005; 26: 319-38.

23.- PELLEGRINO R, VIEGI G, BRUSASCO V, CRAPO $\mathrm{R}$, BURGOS F, CASABURI R, et al. Interpretative strategies for lung function tests. Eur Respir J 2005; 26 : 948-68.

24.- LINARES M, SÁNCHEZ I, CORRALES R, DÍAZ A, ESCOBAR A. Pruebas de función pulmonar en el niño. Rev Chil Pediatr 2000; 71: 228-42.

25.- GUTIÉRREZ M, BEROIZA T, BORZONE G, CAVIEDES I, CÉSPEDES J, GUTIÉRREZ M, et al. Espirometría: Manual de procedimientos. Sociedad Chilena de Enfermedades Respiratorias 2006. Rev Chil Enf Respir 2007; 23: 31-42.

26.- MATTIELLO R, SARRIA E, STEIN R, FISCHER G B, MOCELIN HT, BARRETO S S, et al Functional capacity assessment in children and adolescents with post-infectious bronchiolitis obliterans. J Pediatr (Río Janeiro) 2008; 84: 337-43.

\footnotetext{
Correspondencia a:

Dra. Stefhanie Nayar E.

Unidad Broncopulmonar,

Hospital Roberto del Río, Santiago

E-mail: stefhaniealex@gmail.com
} 